\title{
A Decision Support Tool Based on a Portfolio Selection Model for Crop Planning under Risk
}

\author{
Constanța Zoie RĂDULESCU ${ }^{1}$, Marius RĂDULESCU ${ }^{2}$ \\ ${ }^{1}$ National Institute for Research and Development in Informatics, \\ 8-10 Averescu Avenue, Bucharest 1, RO-011455, ROMANIA, \\ radulescucz@yahoo.com \\ ${ }^{2}$ Institute of Mathematical Statistics and Applied Mathematics, Casa Academiei Române, Calea 13 \\ Septembrie nr.13, Bucharest 5, RO-050711, ROMANIA, \\ mradulescu.csmro@yahoo.com
}

\begin{abstract}
In this paper is presented a decision support tool for crop planning under risk. The software tool is based on a portfolio selection model for crop planning. The portfolio selection model is a minimum financial risk model. It takes into account climate risk and market risk. The decision variable describes the land allocation to crops. The model was solved with the MINLP solver from GAMS. The decision support tool has an interface that facilitates the construction of the input data collection and the user parameters. It gives a flexible way of working and is mainly user oriented. Numerical results obtained with this Decision Support tool are analyzed.
\end{abstract}

Keywords: decision support, software tool, mathematical model, crop planning, risk, portfolio selection.

\section{Introduction}

Computer-based decision support systems (DSSs) have a well-established tradition within crop planning in agriculture. The DSS for crop planning range from simple accounting-based systems to systems based on complex deterministic or stochastic models.

Romanian agriculture, which was for a long time considered a traditional, slow moving economic activity strongly dependent on subsidies and public support, is now a sector affected by many political changes and social pressures derived from environmental concerns and trade liberalizations requirements. Furthermore, the sector dynamics has dramatically increased due to technological innovations, rising world demand for food and raw materials and the new uses of traditional crops as energy sources.

One of the most important decisions faced by farmers is the selection of crops they want to grow. Prospective growers must know how to use risk management strategies in order to select the crops that best suit their needs. One of the most popular approaches to managing risk is to reduce risk exposure through diversification. The uncertainty from the agriculture problems is modeled with the help of probability theory. Many of the practical problems that occur in agriculture are stochastic programming problems with multiple objectives. In practice, in the process of mathematical modeling, one cannot take into account all the factors that have an impact to agricultural production. The number of such factors is large and the growth of their number determines the rapid growth of the complexity of the models. An important mathematical instrument which was successfully applied to modeling the problems from agriculture was portfolio theory. The above mentioned theory was developed as a result of the research in the domain of financial management. The application of portfolio theory for finding an optimal allocation of agricultural land is popular in the literature. The first application of the portfolio theory to crop planning goes back to Freund [6]. In Hardaker [7], Hazell and Norton [8], [9] and Blank [2]-[4] were presented or applied various variants of portfolio theory to the land allocation decisions. In Collender [5], Romero [12]-[14], were studied several models for resources allocation in agriculture that are taking into account specific risks. For other references regarding applications of portfolio theory to agriculture see Radulescu [10], [11].

Our research has focused on the formulation of an original model for crop planning, based on portfolio theory and the design of a decision support software tool based on this model. The paper is organized as it follows. First a crop planning model is proposed. The model is a minimum financial risk model. It considers several classes of land quality, historical data on land productivity and crop market prices. The farmer intends to obtain optimal production plans that minimize the financial risk. A decision support software tool based on 
this model is described in our paper. The model was solved with the MINLP solver from GAMS. The software application has an interface that facilitates the construction of the input data collection and the user parameters. It gives a flexible way of working and is mainly user oriented. A practical example is given before the presentation of the paper conclusions.

\section{A Minimum Financial Risk Model}

In the following is formulated a 0-1 integer programming model for crop planning in agriculture. The model takes into account weather risks and market risks. Input data include historical data on land productivities for various crops and soil types.

Consider a farm which has an agricultural land divided into several plots $P_{1}, P_{2}, \ldots, P_{m}$. We consider that if a plot is cultivated then it is cultivated with the same crop. Also we consider that the soil quality of a plot is homogeneous.

Denote by $S_{j}$ the area of the plot $P_{j}, j=1,2, \ldots$, $m$. We consider that the farmer have to choose a crop plan from $n$ crops $C_{1}, C_{2}, \ldots, C_{n}$, that is to make an allocation of crops to plots.

Consider the probability space $(\Omega, \mathrm{K}, \mathrm{P})$. Denote $I=\{1,2, \ldots, n\}, J=\{1,2, \ldots, m\}$. For every $i \in I, \quad j \in J$ we define the random variables $c_{i j}: \Omega \rightarrow \mathbf{R}_{+}$and $b_{i}: \Omega \rightarrow \mathbf{R}_{+} \cdot c_{i j}(\cdot)$ are called the plot productivity functions and $b_{i}$ are called the market price functions. $c_{i j}(\cdot)$ is the quantity of crop $C_{i}$ that can be produced on an area of one hectare of plot $P_{j}$ and $b_{i}$ is the market price for a quantity of one $\mathrm{kg}$ of crop $C_{i}$. For every $i \in I, j \in J$, denote by:

- $a_{i j}$ - the cost for cultivating one ha of the plot $P_{j}$ with crop $C_{i}$

- $\quad c_{i j}$ - the plot productivity coefficient, that is the quantity of crop $C_{i}$ obtained from an area of one hectare of plot $P_{j}$.

- $x_{i j}$ - the decision variable that takes the value 1 if the crop $C_{i}$ is cultivated on plot
$P_{j}$ and takes value zero if the crop $C_{i}$ is not cultivated on the plot $P_{j}$.

- $\left[M_{1}, M_{2}\right]$ the range for the sum of money available for investment

- $Q_{i}$ the lower bound for the yield of crop $C_{i}$ necessary to be obtained (contractual levels)

The yield of crop $C_{i}$ is equal to $\sum_{j=1}^{m} c_{i j}(\cdot) S_{j} x_{i j}$, $i \in I$. The cultivation cost when the land allocation $\mathbf{x}=\left(x_{i j}\right)$ of crops to plots is applied is equal to $\sum_{i=1}^{n} \sum_{j=1}^{m} a_{i j} S_{j} x_{i j}$.

The constraints $\sum_{i=1}^{n} x_{i j} \leq 1, \quad j \in J$ show that every plot is cultivated with at most one crop. The return (respectively the expected return) obtained when the decision matrix $\mathbf{x}$ is used is equal to:

$\psi(\cdot, \mathbf{x})=\sum_{i=1}^{n} \sum_{j=1}^{m} b_{i} c_{i j} S_{j} x_{i j}$ (respectively)

$E[\psi(\cdot, \mathbf{x})]=\sum_{i=1}^{n} \sum_{j=1}^{m} E\left(b_{i} c_{i j}\right) S_{j} x_{i j}$.

For every $i, \alpha \in I, j, \beta \in J$, denote $\rho_{i j \alpha \beta}=E\left[b_{i} c_{i j} b_{\alpha} c_{\alpha \beta}\right\rfloor-E\left\lfloor b_{i} c_{i j}\right\rfloor \cdot E\left\lfloor b_{\alpha} c_{\alpha \beta}\right\rfloor$.

Then the variance of the return is equal to $\operatorname{Var}(\psi(\cdot, \mathbf{x}))=\sum_{i=1}^{n} \sum_{j=1}^{m} \sum_{\alpha=1}^{n} \sum_{\beta=1}^{m} \rho_{i j \alpha \beta} X_{i j} x_{\alpha \beta} S_{j} S_{\beta}$. The variance of the return has the meaning of financial risk.

In the frame of this problem the farmer tries to find an optimal allocation of crops to plots that minimize the financial risk taking into account that the expected return is greater than a given level $W$.

$\left\{\begin{array}{l}\min (\operatorname{Var}(\psi(\cdot, \mathbf{x}))) \\ \sum_{j=1}^{m} E\left(c_{i j}\right) S_{j} x_{i j} \geq Q_{i}, i \in I \\ \sum_{i=1}^{n} \sum_{j=1}^{m} E\left(b_{i} c_{i j}\right) S_{j} x_{i j}-\sum_{i=1}^{n} \sum_{j=1}^{m} a_{i j} S_{j} x_{i j} \geq W \\ M_{1} \leq \sum_{i=1}^{n} \sum_{j=1}^{m} a_{i j} S_{j} x_{i j} \leq M_{2} \\ \sum_{i=1}^{n} x_{i j} \leq 1, \text { for every } j \in J, \\ x_{i j} \in\{0,1\}, i \in I, j \in J\end{array}\right.$ 
An important problem in the decision process of the choice of a crop allocation to plots is the existence of some indicators that will guide the farmer in the selection of the parameters of the problem: $M_{1}, M_{2}$ and $W$. These indicators can be computed by solving several binary programming problems.

Denote by $M_{1}^{\min }$ (respectively by $M_{2}^{\max }$ ) the optimal value of the following two binary programming problems:

$\left\{\begin{array}{l}\min \left(\sum_{i=1}^{n} \sum_{j=1}^{m} a_{i j} S_{j} x_{i j}\right) \\ \sum_{j=1}^{m} E\left(c_{i j}\right) S_{j} x_{i j} \geq Q_{i}, i \in I \\ \sum_{i=1}^{n} x_{i j} \leq 1, \text { for every } j \in J, \\ x_{i j} \in\{0,1\}, i \in I, j \in J\end{array}\right.$

and

$$
\left\{\begin{array}{l}
\max \left(\sum_{i=1}^{n} \sum_{j=1}^{m} a_{i j} S_{j} x_{i j}\right) \\
\sum_{j=1}^{m} E\left(c_{i j}\right) S_{j} x_{i j} \geq Q_{i}, i \in I \\
\sum_{i=1}^{n} x_{i j} \leq 1, \text { for every } j \in J, \\
x_{i j} \in\{0,1\}, i \in I, j \in J
\end{array}\right.
$$

The farmer has to choose the parameters $M_{1}, M_{2}$ in the range $\left[M_{1}^{\min }, M_{2}^{\max }\right]$. After that the farmer has to determine the range from where he has to choose the parameter $W$. Denote by $W_{1}$ (respectively by $W_{2}$ ) the optimal value of the following binary programming problems:

$$
\left\{\begin{array}{l}
\min \left(\sum_{i=1}^{n} \sum_{j=1}^{m} E\left(b_{i} c_{i j}\right) S_{j} x_{i j}-\sum_{i=1}^{n} \sum_{j=1}^{m} a_{i j} S_{j} x_{i j}\right) \\
\sum_{j=1}^{m} E\left(c_{i j}\right) S_{j} x_{i j} \geq Q_{i}, i \in I \\
M_{1} \leq \sum_{i=1}^{n} \sum_{j=1}^{m} a_{i j} S_{j} x_{i j} \leq M_{2} \\
\sum_{i=1}^{n} x_{i j} \leq 1, \text { for every } j \in J, \\
x_{i j} \in\{0,1\}, i \in I, j \in J
\end{array}\right.
$$

and

$$
\left\{\begin{array}{l}
\max \left(\sum_{i=1}^{n} \sum_{j=1}^{m} E\left(b_{i} c_{i j}\right) S_{j} x_{i j}-\sum_{i=1}^{n} \sum_{j=1}^{m} a_{i j} S_{j} x_{i j}\right) \\
\sum_{j=1}^{m} E\left(c_{i j}\right) S_{j} x_{i j} \geq Q_{i}, i \in I \\
M_{1} \leq \sum_{i=1}^{n} \sum_{j=1}^{m} a_{i j} S_{j} x_{i j} \leq M_{2} \\
\sum_{i=1}^{n} x_{i j} \leq 1, \text { for every } j \in J, \\
x_{i j} \in\{0,1\}, i \in I, j \in J
\end{array}\right.
$$

The farmer has to choose the parameter $W$ in the interval $\left[W_{1}, W_{2}\right]$.

\section{A Decision Support Tool for Crop Planning under Risk}

Based on the crop planning model presented above, a decision support software tool was developed. Its name is MINRISK. Some of the optimization modules from our software application are based on the theory developed in Andrei [1]. The crop planning model in MINRISK was solved with the solver MINLP from GAMS. MINRISK is composed from the following modules: "User Interface", "Input data", "Model resolution”, and "Sensitivity analysis".

The input data collection is built and validated in the module "Input data" following a set of rules.

The module "model resolution" facilitates the model building, transition of the model to the GAMS solver MINLP, solves the model and returns the results back. In order to make the validation of the user parameters the module solves several mathematical programming problems. As a result, ranges of variation for the user parameters are determined. For example, for the determination of the range of variation of the user parameter $W$, the module solves two linear programming problems.

The results are visualized and saved according to the user demand. The module "sensitivity analysis" allows by the variation of the user parameter $W$, the computation of the efficient frontier of the crop planning model. The module "User interface" gives a flexible way of working, user oriented.

The decision support software tool MINRISK was realized in Visual Basic Net. The system architecture is presented in Figure 1. 


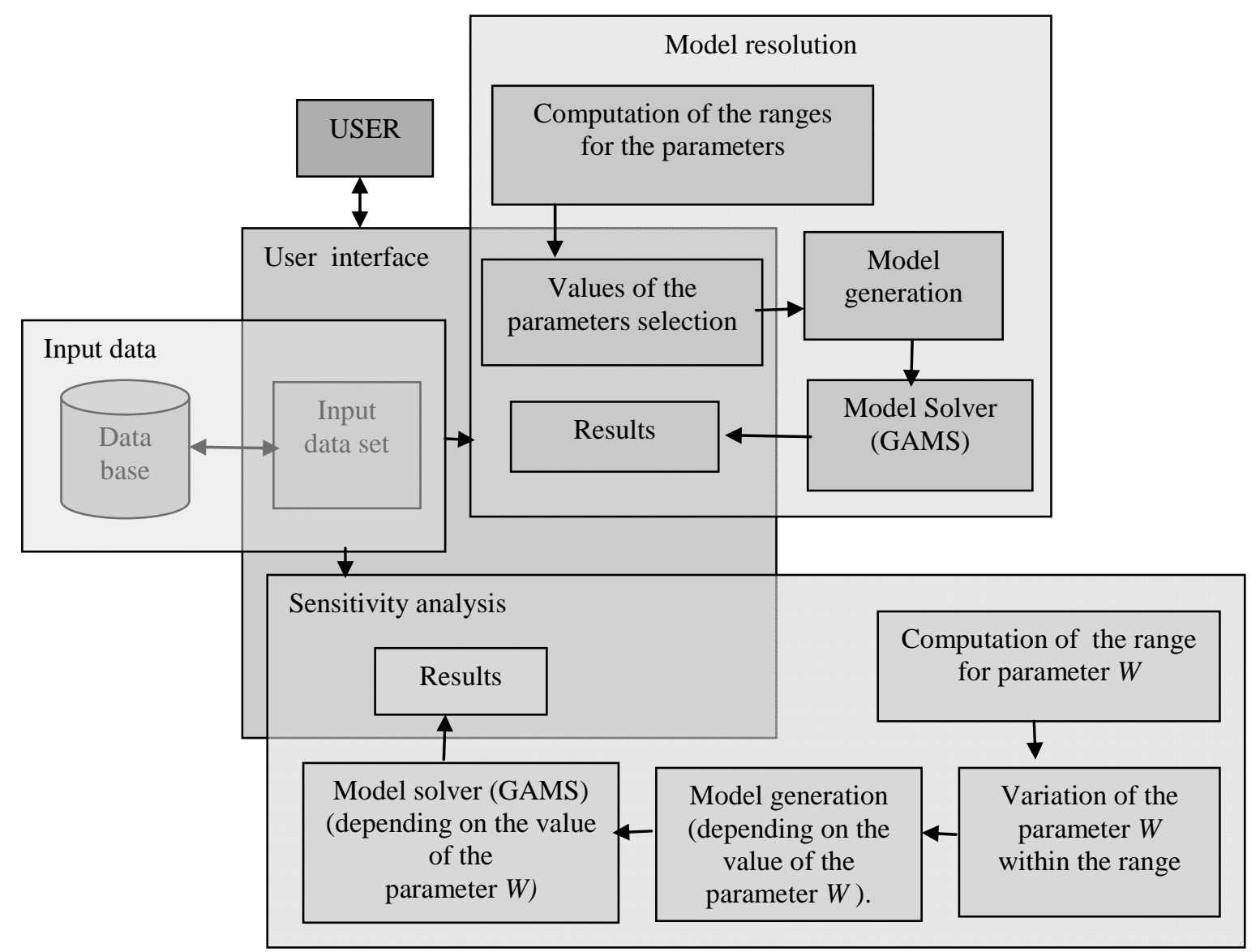

Figure 1. The system architecture of the software application

The input data for the model are:

- The set of crops

- The set of available plots for cultivation

- The period that is taken into account

- Historical data on market prices for crops

- Historical data on crop productivities on plots

- Cultivation cost (euro per ha) for each crop and plot

- The areas of plots

- Minimum quantities (quotas) for each crop

The user parameters are:

- Minimum and the maximum value for the amount invested by the farmer (parameters $\left.M_{1}, M_{2}\right)$

- Minimum profit that farmer wants to obtain (parameter W)

The decision variable is the allocation matrix $\mathbf{x}=\left(x_{i j}\right)$ whose entries are 0 and 1 .

The output variables are:

- Optimal allocation of crops to plots
- The quantities of each crop that should be cultivated in the case the allocation is optimal (That is when the financial risk has a minimum value)

- The return obtained as the result of application of the optimal allocation of crops

- The sum invested for the optimum crop plan.

The constraints in the model are:

- The average quantities from each crop are greater than the minimal quotas

- The sum available for investment is in the range $\left[M_{1}, M_{2}\right]$ specified by the farmer

- On each plot is cultivated at most one crop

- The expected return is greater or equal than the user parameter $\mathrm{W}$

\section{Numerical Example}

We consider six crops and eight plots of cultivated land. The crops are $\mathrm{C}_{1}=$ wheat, $\mathrm{C}_{2}=$ rye, $\mathrm{C}_{3}=$ barley, $\mathrm{C}_{4}=$ oats, $\mathrm{C}_{5}=$ maize, $\mathrm{C}_{6}=$ sunflower. 
The areas of the plots are $S_{1}=10 \mathrm{ha}, S_{2}=12 \mathrm{ha}$, $S_{3}=7$ ha, $S_{4}=11$ ha, $S_{5}=14$ ha, $S_{6}=16$ ha, $S_{7}=9$ ha and $S_{8}=12$ ha. The years for historical data are 2001 - 2007. Quantities (quotas) minimum for each crop are $\mathrm{Q}_{1}=10000 \mathrm{~kg}$ wheat, $\mathrm{Q}_{2}=$ $12000 \mathrm{~kg}$ rye, $\mathrm{Q}_{3}=12000 \mathrm{~kg}$ barley, $\mathrm{Q}_{4}=$ $10000 \mathrm{~kg}$ oats, $\mathrm{Q}_{5}=20000 \mathrm{~kg}$ maize and $\mathrm{Q}_{6}=$ $10000 \mathrm{~kg}$ sunflower.

The data are from a agricultural farm in the South Muntenia Region of Romania.

The choice of user parameters is presented in Figure 2. In the first box of the first row is displayed $M_{1}^{\min }$ while in the second box is displayed $M_{1}$. In the first box of the second row is displayed $M_{2}$ while in the second box is displayed $M_{2}^{\max }$. Note that $M_{1}^{\min }$ and $M_{2}^{\max }$ are computed while $M_{1}$ and $M_{2}$ are chosen by the farmer.

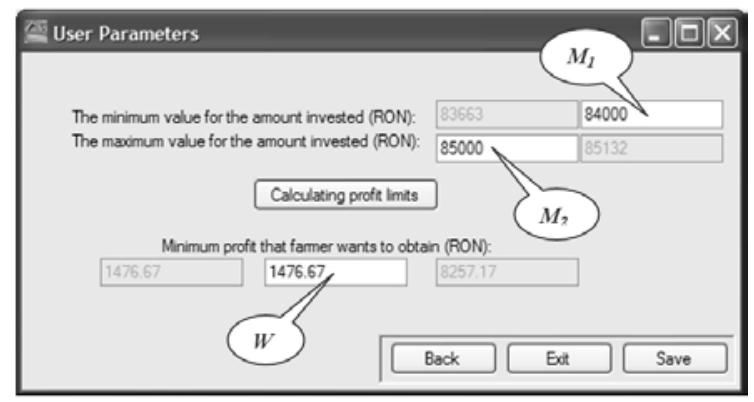

Figure 2. The choice of user parameters $M_{1}, M_{2}$ and $\mathrm{W}$

The optimal solution, allocation of crops on plots, is presented in Figure 3 in two formats. In the first format the optimal solution is presented in a matrix of crops / plots. In the second format the optimal solution is presented in a vector. In Figure 3 is also displayed the minimum value of the risk.

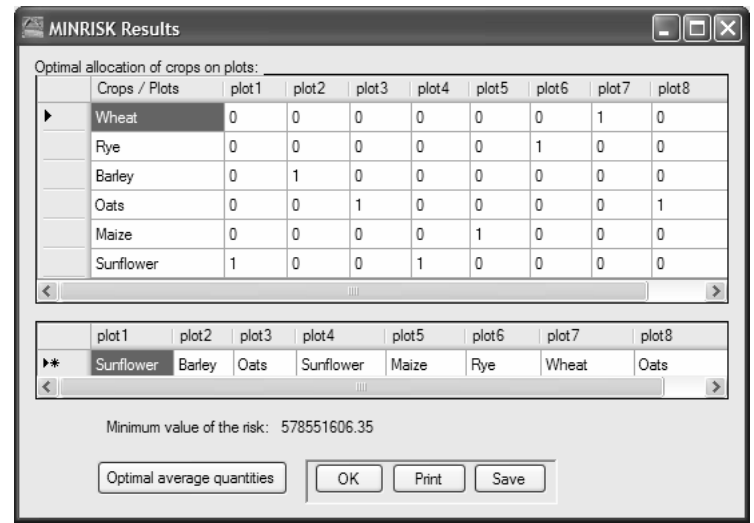

Figure 3. The optimal solution - allocation of crops on plots
The efficient frontier of the minimum financial risk problem is displayed in Figure 4.

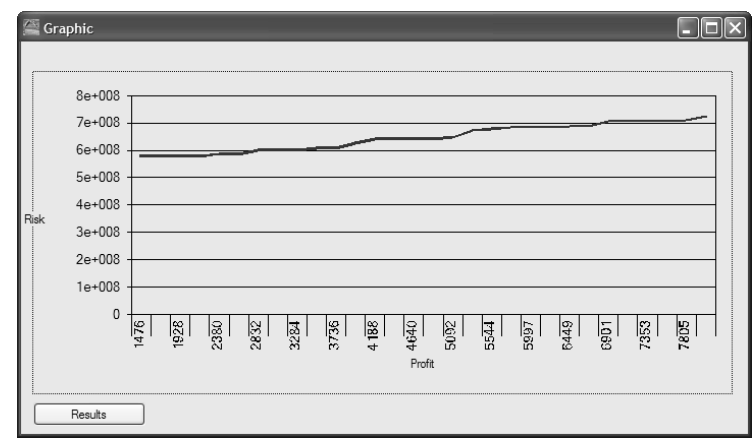

Figure 4. The efficient frontier of the minimum financial risk problem

The efficient frontier is the graph of the map that associates to values of $W$ the optimal value of the objective map of the minimum financial risk problem.

The numerical results are obtained with the software tool MINRISK.

\section{Conclusion}

Our research has been focusing on the formulation of a minimum risk model for crop planning, based on portfolio theory and the design of a software tool based on this model. In order to facilitate the integration of the software tool in a decision support system the range of variation for the parameters of the model were computed. The model was solved with the MINLP solver from GAMS. A numerical result is analyzed and several captures of the screens from the software tool are displayed.

\section{Acknowledgements}

This work was supported by a grant of the Romanian National Authority for Scientific Research, CNCS - UEFISCDI, project number PN-II-ID-PCE-2011-3-0908.

\section{REFERENCES}

1. ANDREI, N., On Quadratic Internal Model Principle in Mathematical Programming, Studies in Informatics and Control, Volume 18, Number 4, 2009, pp.337-348.

2. BLANK, S. C., Returns to Limited Crop Diversification. Western Journal of 
Agricultural Economics, no. 15, 1990, pp. 204-212.

3. BLANK, S. C., C. A. CARTER, J. McDONALD, Is the Market Failing Agricultural Producers Who Wish to Manage Risks?, Contemporary Economic Policy, no.15, 1997, pp.103-112.

4. BLANK, S. C., Producers Get Squeezed Up the Farming Food Chain: A Theory of Crop Portfolio Composition and Land Use. Review of Agricultural Economics, no. 23, 2001, pp. 404-422.

5. COLLENDER, R. N., Estimation Risk in Farm Planning under Uncertainty. American Journal of Agricultural Economics, no. 71, 1989, pp. 996-1002.

6. FREUND, R. J., The Introduction of Risk into a Programming Model. Econometrica, no. 24, 1956, pp. 253-263.

7. HARDAKER, J. B., R. B. M. HUIRNE, J. R. ANDERSON, G. LIEN, Coping with Risk in Agriculture, 2nd ed., CABI Publishing, Oxfordshire, 2004.

8. HAZELL, P. B. R, A Linear Alternative to Quadratic and Semivariance Programming for Farm Planning under Uncertainty. American Journal of Agricultural Economics, no. 53, 1971, pp. 53-62.
9. HAZELL, P. B. R., R. D. NORTON, Mathematical Programming for Economic Analysis in Agriculture, Macmillan, New York, 1986.

10. RADUlESCU, M., S. RADULESCU, C. Z. RADULESCU, Mathematical Models for Optimal Asset Allocation, (Romanian). Editura Academiei Române, Bucureşti, 2006.

11. RĂDULESCU, M., C. Z. RĂDULESCU, Ghe. ZBĂGANU, A Portfolio Theory Approach to Fishery Management, Studies in Informatics and Control, Vol. 19, Nr. 3, 2010, pp.285-294.

12. ROMERO, C., Una aplicación del modelo de Markowitz a la selección de planes de variedades de manzanos en la provincia de Lérida. Revista de Estudios Agro-sociales, no. 97, 1976, pp. 61-79.

13. ROMERO, C., T. REHMAN, Multiple Criteria Analysis for Agricultural Decisions, Second Edition, Volume 11 (Developments in Agricultural Economics) Elsevier, Amsterdam, 2003.

14. ROMERO, C., Risk Programming for Agricultural Resource Allocation: A Multidimensional Risk Approach. Annals of Operations Research, 94, 2000, pp. 57-68. 